\title{
Jet lag: life in the fast (and feast) lane
}

\author{
Stephen B Hanauer
}

Travel to distant places to share in the expertise of one's field is seen as a perk of academic medicine. I've developed a personalized routine to help me handle a frequent complication of traversing numerous time zones-jet lag. However, I'm afraid that the feelings of irritability and general disorientation associated with jet lag have been only minimally abated by exercising on arrival at my destination, my enjoyment of foreign cuisines (and wine) and benzodiazepine-aided sleep.

I must admit that I have never subscribed to any anti-jet-lag mythologies and/or methodologies, owing to their inadequate evidencebase, and have generally had success in letting espresso and adrenaline take over for shortterm activities such as lectures or advisory board meetings. However, a website licensed and managed for the US Department of Energy by the University of Chicago Argonne National Laboratory (http://www.antijetlagdiet.com) has led me to rethink my strategies for coping with travel. The diet plan and activity-sleep schedule reported on the website is ascribed to the efforts of a biologist, Dr Charles Ehret, who studied circadian rhythms that are controlled by a so-called molecular clock at a cellular level. Before travel, the plan advocates alternating days of feasting on highprotein foods with days of relative fasting on small portions of low-carbohydrate foods. The schedule culminates with a fast before arrival and a high-protein feast eaten at the appropriate time for breakfast at the end destination. To my knowledge, the only clinical trial to test this diet plan successfully reduced symptoms of jet lag in military personnel who traveled

\section{...from cells \\ to organisms, \\ it seems that \\ those in the \\ 'fast' lane \\ may develop \\ adaptive \\ and survival \\ advantages.}

SB Hanauer is Editorin-Chief of Nature Clinical Practice Gastroenterology \& Hepatology.

\section{Competing interests \\ The author declared no} competing interests.

www.nature.com/clinicalpractice doi:10.1038/ncpgasthep1187 from the US to Korea and back (Military Med [2002] 167: 451-453).

This year, Saper and colleagues report their use of molecular genetics to investigate the impact of light-dark cycles and food availability on circadian rhythms (Science [2008] 320: 1074-1077). These investigators begin with the observations that, "When food is plentiful, circadian rhythms of animals are powerfully entrained by the light-dark cycle." but when food access is restricted to periods during their sleep cycle animals "... will shift most of their circadian rhythms to match the food availability." By using viral vectors to insert the clock gene Bmal1 into targeted areas of the brain of mice, Saper and colleagues were able to separate light-entrainable from food-entrainable circadian rhythms. These findings suggest that it is possible to re-regulate circadian rhythms and also provide bedside-tobench support for the feast and fast program advocated in the anti-jet-lag diet.

Fasting is also known to have an anti-aging effect in animals. Studies in mice have also suggested that fasting for 2 days protects healthy cells against high-dose chemotherapy and leads to prolonged survival (Proc Natl Acad Sci USA [doi:10.1073/pnas.0708100105]). Short periods of starvation may also make normal human cells more resistant to chemotherapy, in contrast to cancerous brain cell lines.

Thus, from cells to organisms, it seems that those in the 'fast' lane may develop adaptive and survival advantages. Of course, supportive clinical trials are needed to confirm the timing, dose and duration of feast and fast cycles necessary to optimize individual outcomes. 